\section{Microscopy Coming Events}

\section{7}

New Zealand Conference on Microscopy January 31-February 3, 2017

Auckland, New Zealand

www.microscopy2017.co.nz

\author{
AMAS XIV - 14th Australian Microbeam \\ Biennial Symposium \\ February 6-10, 2017 \\ Brisbane, Australia \\ http://microscopy.org.au/amas/
}

Biophysical Society 61st Annual Meeting February 11-15, 2017

New Orleans, LA

www.biophysics.org/Meetings/AnnualMeeting/Future/ tabid/495/Default.aspx

Pittcon 2017 Conference \& Expo

March 5-9, 2017

Chicago, IL

http://pittcon.org/pittcon-2017

Novel Techniques in Microscopy

April 2-5, 2017

San Diego, CA

www.osa.org/en-us/meetings/global calendar/events/

novel_techniques_in_microscopy_\% $281 \% 29$

253rd ACS National Meeting \& Exposition April 2-6, 2017

San Francisco, CA

www.acs.org/content/acs/en/meetings/nationalmeetings/ meetings.html

Microscopy \& Microanalysis 2017

August 6-10, 2017

St. Louis, MO

www.microscopy.org

\section{8}

Microscopy \& Microanalysis 2018

August 5-9, 2018

Baltimore, MD

www.microscopy.org

\section{9}

Microscopy \& Microanalysis 2019

August 4-8, 2019

Portland, OR

www.microscopy.org

\section{0}

Microscopy \& Microanalysis 2020

August 2-6, 2020

Milwaukee, W

www.microscopy.org

\section{1}

Microscopy \& Microanalysis 2021

August 1-5, 2021

Pittsburgh, PA

www.microscopy.org

More Meetings and Courses Check the complete calendar near the back of this magazine.

\title{
Two-Way Traffic Determined by Different Tracks in Cilia
}

\author{
Stephen W. Carmichael and Jeffery L. Salisbury \\ Mayo Clinic, Rochester, MN 55905
}

carmichael.stephen@mayo.edu, salisbury.jeffery@mayo.edu

The cilium and the flagellum are conserved organelles that play fundamental roles in cellular signaling, sensing, and motility. Cilia and flagella have a complex microtubule-based axoneme that includes nine peripheral microtubule doublets, each comprised of a complete A-tubule and an incomplete B-tubule. However, the function of this distinctive geometry has remained unknown until now. In a recent study, Ludek Stepanek and Gaia Pigino use the model organism Chlamydomonas to reveal a new understanding of the functional significance of the A- and B-tubule structure [1].

It is known that ciliary microtubule doublets function as "railways" for intraflagellar transport (IFT), the process required for the assembly and disassembly of cilia. Large protein complexes, known as IFT trains, rapidly traverse up and down the cilium to move ciliary "building blocks" between the cell body and the distal tip of the cilium where the assembly of the cilium occurs. Electron microscopy (EM) has shown that the IFT trains move along the doublets. However, it has not been clear how the railway is organized to avoid collisions between anterograde and retrograde trains.

Stepanek and Pigino used total internal fluorescence (TIRF) microscopy to show that trains tagged with green fluorescent protein interacted with each other in both the anterograde and retrograde directions. However, trains headed in the opposite direction did not interact, suggesting
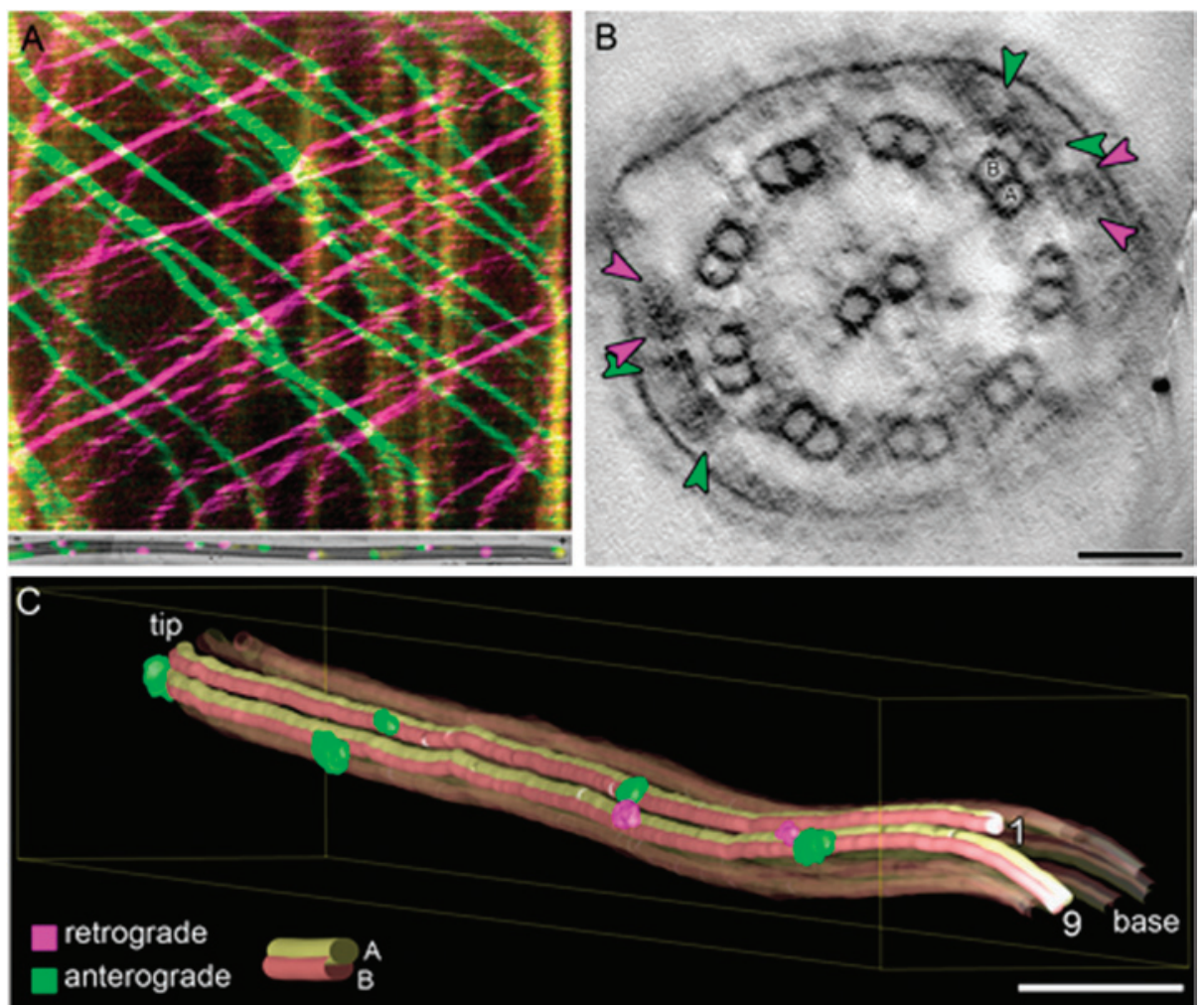

Figure 1: Anterograde and retrograde IFT trains use different microtubules in the same doublet. (A) Kymograph showing directional IFT train movement during live-cell imaging of Chlamydomonas (IFT27-GFP strain). In this kymograph a green particle moving in the anterograde direction tracks a line from the upper left to the lower right; similarly for a magenta particle traveling retrograde from upper right to lower left. Vertical drop indicates distance traveled, and slope indicates rate of movement. (B) Virtual tomogram slice, showing cross-sectional view of anterograde trains (green arrowheads) and retrograde trains (magenta arrowheads) stopped next to each other on the same doublet. Scale bar $=50 \mathrm{~nm}$. (C) Segmentation of an axoneme showing anterograde trains (green) and retrograde trains (magenta) moving simultaneously on microtubule doublet 9 . Scale bar $=25 \mathrm{~nm}$. 


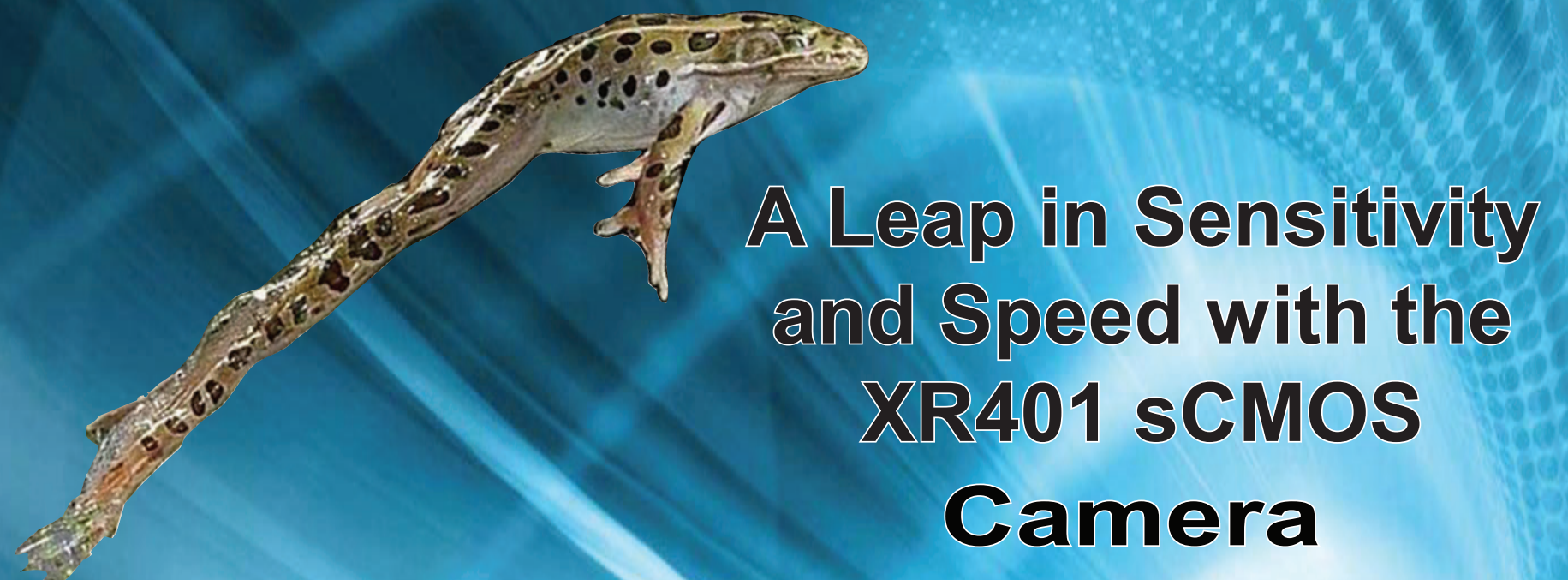

- Cryo TEM

- Low Dose TEM

- Diffraction

- In-Situ TEM

- Extraordinary speed

- Non-Blooming Sensor $0<2$ e/pixel noise

Adenovirus

Dr. Cameron Ackerley

The Hospital for Sick Children

\section{A Leap in Sensitivity and Speed with the XR401 sCMOS Camera}
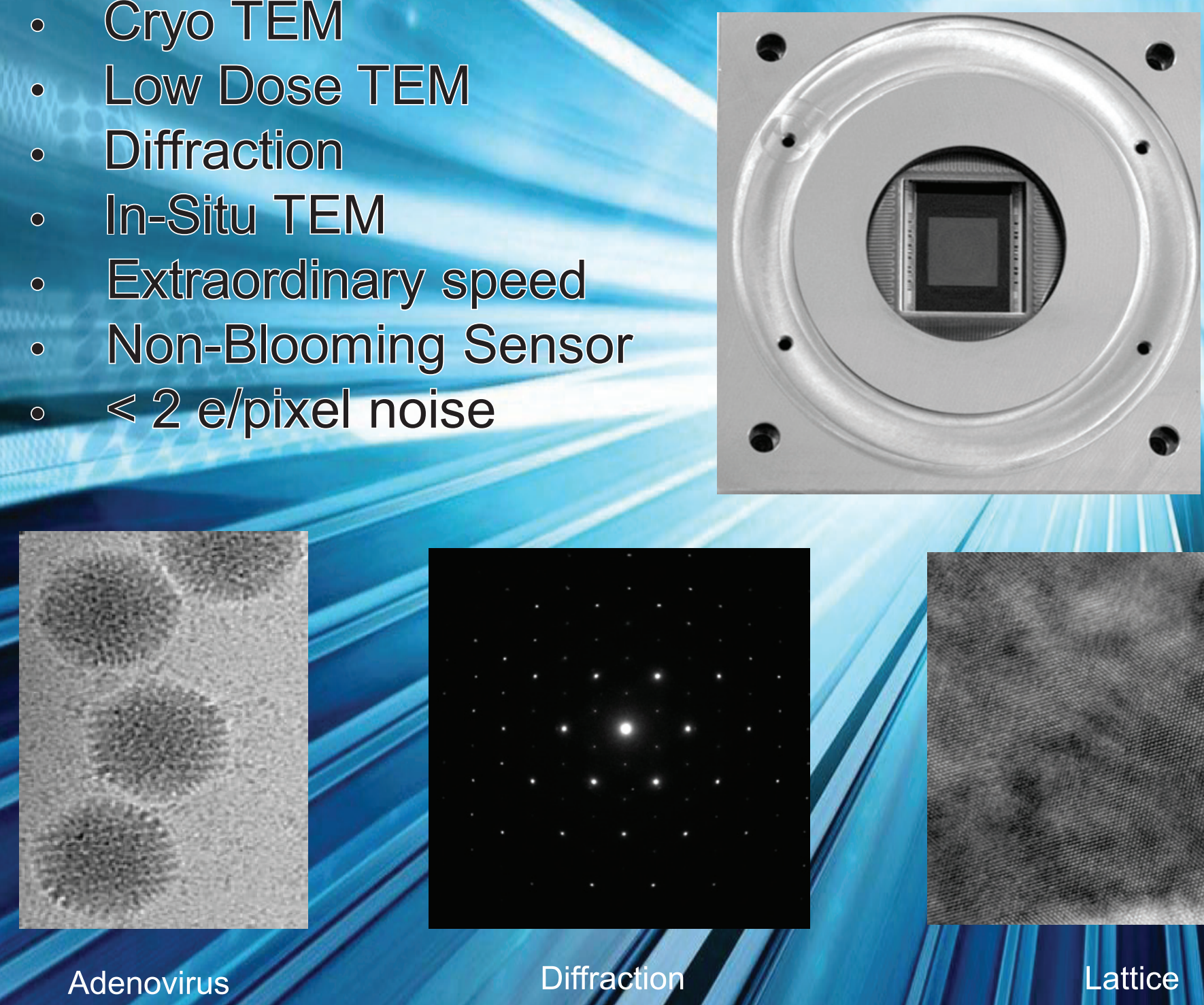

Dr. Pengfei Hu at the Shanghai University

Lattice 
that a mechanism must exist to prevent collisions. Because TIRF microscopy does not provide sufficient resolution to demonstrate a relationship between trains and microtubules, the authors took the correlative light and EM (CLEM) approach. In order to provide sufficient spatiotemporal resolution to analyze IFT trains, they developed a new method to provide millisecond resolution in correlative TIRF and three-dimensional (3D) EM. The experimental cell was rapidly fixed with glutaraldehyde during time-lapse TIRF imaging of IFT trains. The position of each fixed IFT train was cross-referenced to its direction of movement just before fixation. The same cell was then prepared for EM tomography. They obtained sufficient spatial resolution to observe the structural details of each train and its interaction with microtubules. In this manner they identified anterograde and retrograde trains unambiguously, as well as trains that remained stationary (Figure 1).

Using these and other techniques Stepanek and Pigino showed that each microtubule doublet is used as a bidirectional double-track railway with anterograde IFT trains moving toward the ciliary tip along B-microtubules and retrograde trains moving toward the cell body along A-microtubules. Because A- and B-microtubules are thought to share the same tubulin arrangement, they suggested that IFT trains recognize specific post-translational modifications on A- versus B-microtubules. Thus, the microtubule doublet geometry provides direction-specific rails to coordinate bidirectional transport of ciliary and flagellar components. At the very least, this study highlights the critical role played by microtubule doublets in the assembly of cilia and flagella.

\section{References}

[1] L Stepanek and G Pigino, Science 352(6281) (2016) 721-24.

[2] The authors gratefully acknowledge Dr. Gaia Pigino for reviewing this article.

VT

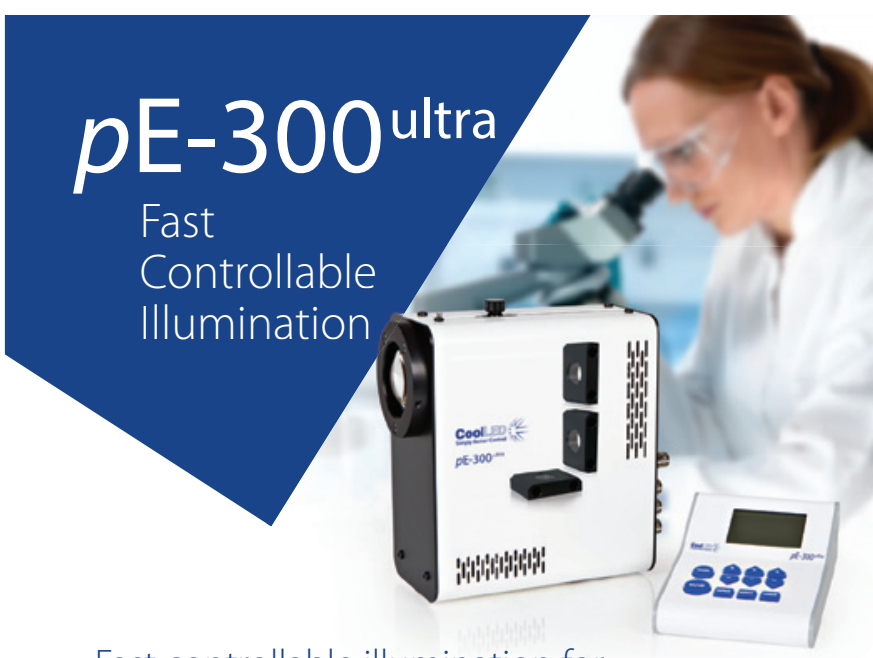

Fast controllable illumination for:

Optogenetics

Electrophysiology

High speed microscopy applications Cool [L

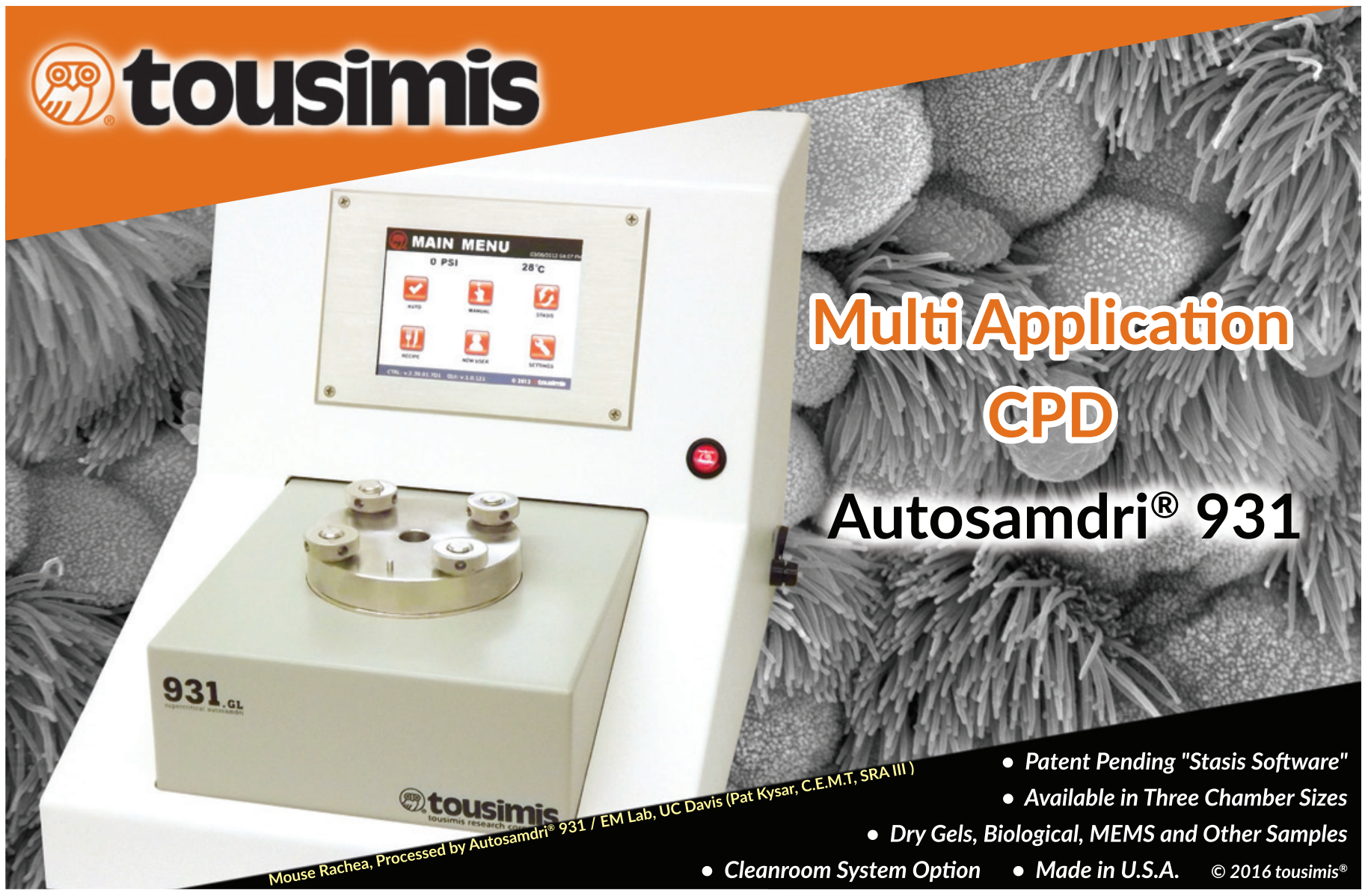




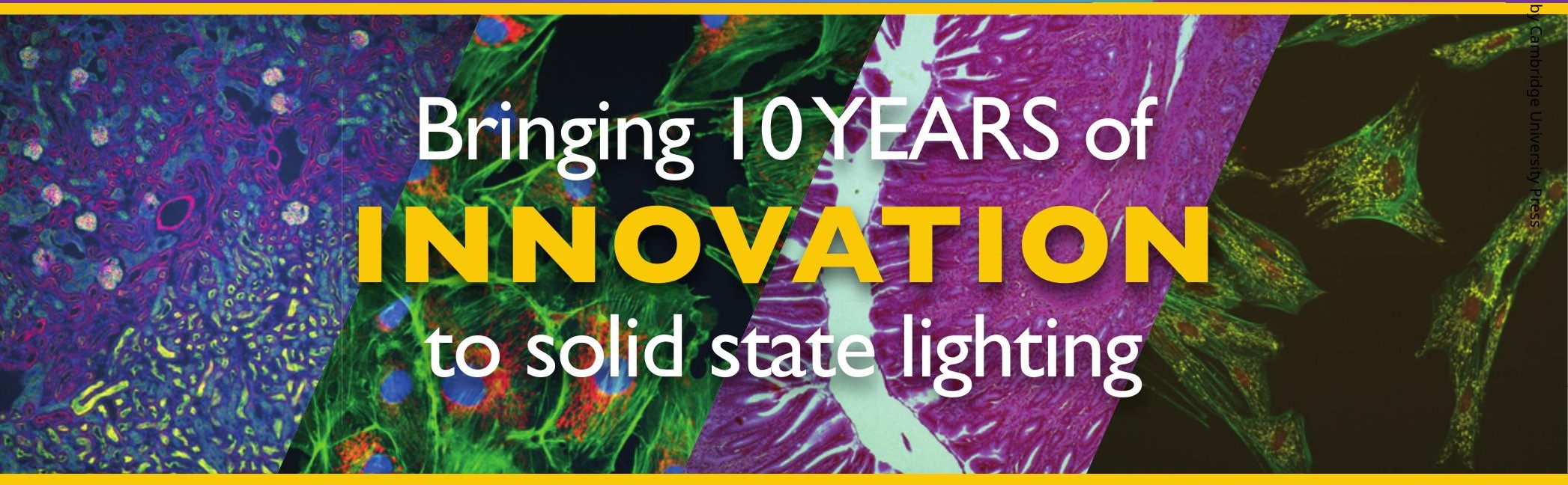

\section{FREE Control Pod*}

As a thank you to our loyal customers, Lumencor is offering a free control pod with every electronically controlled light engine purchase in 2017. SOLA SE light engine ${ }^{\circledR}$, SPECTRA X light engine ${ }^{\circledR}$ and LIDA light engine ${ }^{\circledR}$ models apply.

*End user customers only. Equipment manufacturers do not apply.
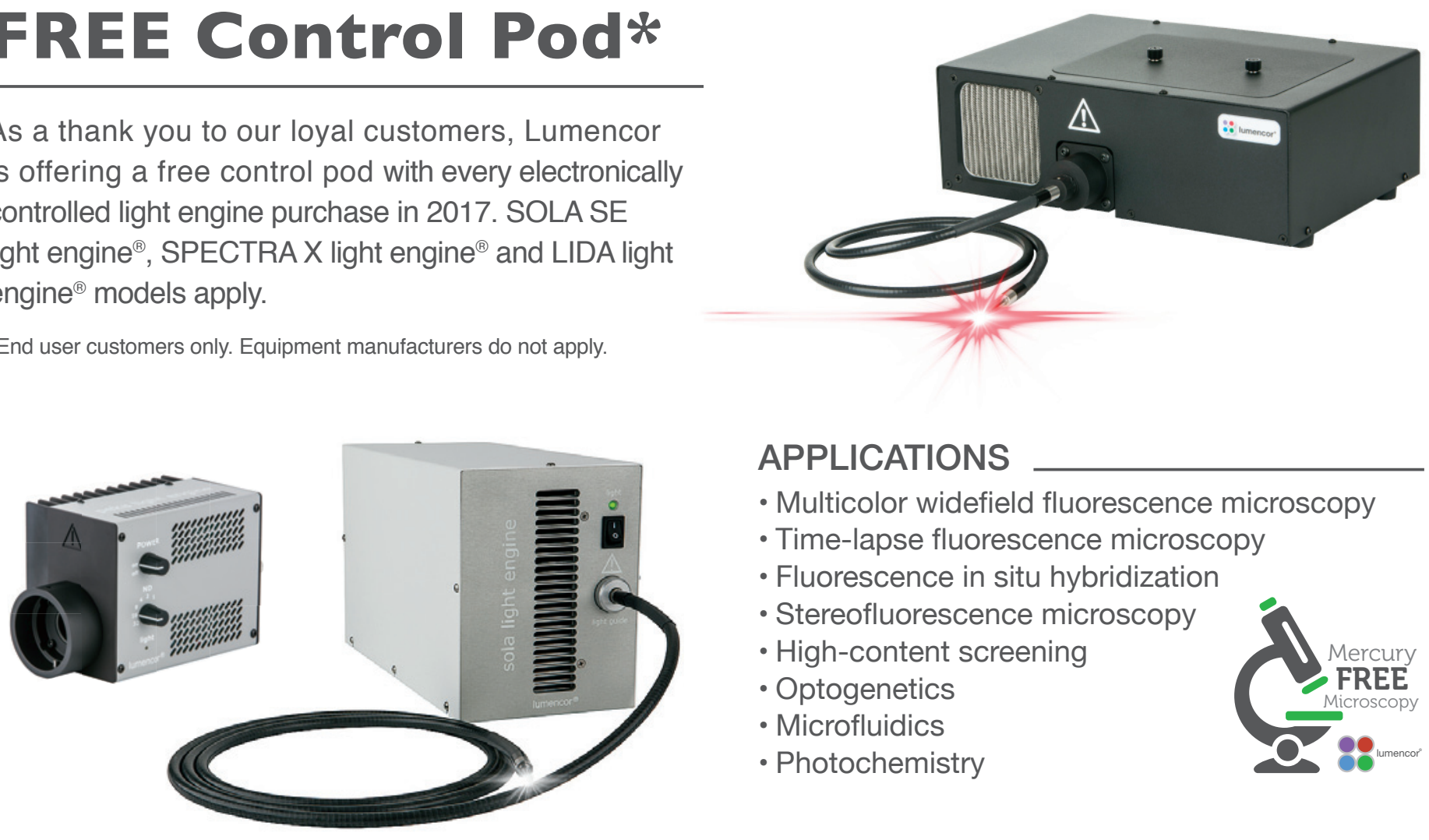

APPLICATIONS

- Multicolor widefield fluorescence microscopy

- Time-lapse fluorescence microscopy

- Fluorescence in situ hybridization

- Stereofluorescence microscopy

- High-content screening

- Optogenetics

- Microfluidics

- Photochemistry

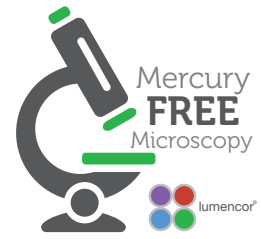

